\begin{tabular}{|c|l|}
\hline Title & Theory of thermal boundary resistance between small particles and liquid helium. II. Normal liquid 3He \\
\hline Author(s) & Nishiguchi, Norihiko; Nakayama, Tsuneyoshi \\
\hline Citation & $\begin{array}{l}\text { Physical Review B, 25(9), 5720-5728 } \\
\text { https://doi.org/10.1103/PhysRevB.25.5720 }\end{array}$ \\
\hline Issue Date & 1982-05-01 \\
\hline Doc URL & http://hdl.handle.net/2115/47090 \\
\hline Rights & ○ 1982 The A merican Physical Society \\
\hline Type & article \\
\hline File Information & PhysRevB.25.5720.pdf \\
\hline
\end{tabular}

Instructions for use 


\title{
Theory of thermal boundary resistance between small particles and liquid helium. II. Normal liquid ${ }^{3} \mathrm{He}$
}

\author{
Norihiko Nishiguchi and Tsuneyoshi Nakayama \\ Department of Engineering Science, Hokkaido University, Sapporo 060, Japan
}

(Received 26 October 1981)

\begin{abstract}
The previous theory on the thermal boundary resistance between small particles and liquid He II [Phys. Rev. B 24, 6421 (1981)] is extended to the case for normal liquid ${ }^{3} \mathrm{He}$ in terms of Landau Fermi-liquid theory. It is shown that the toroidal-mode phonons in small particles contribute to the heat flow through the excitation of transverse zero sound in contrast to the case of liquid He II. The calculated resistances exhibit a $T^{-3}$ behavior above some temperature which depends on the size and elastic property of the small particle. In this temperature regime, the calculated results for silver and copper particles agree well with the observed resistances in both magnitude and temperature dependence. At lower temperatures the theory predicts exponentially increasing resistances with decreasing temperature as in the case for liquid $\mathrm{He}$ II. The relative contributions to the heat flow due to longitudinal and transverse zero sound are obtained as a function of temperature.
\end{abstract}

\section{INTRODUCTION}

The heat transfer between liquid $\mathrm{He}$ II and a solid produces a temperature discontinuity at the interface of the two media. This phenomenon, which is referred to as thermal boundary resistance, was first discovered for liquid $\mathrm{He}$ II by Kapitza. ${ }^{1}$ A similar temperature discontinuity also appears at the boundary of normal liquid ${ }^{3} \mathrm{He}$ and a solid, ${ }^{2}$ and it is identified that the thermal resistance is not peculiar to superfluid ${ }^{4} \mathrm{He}$. In experiments on the heat exchange below about $100 \mathrm{mK}$, solids have been often used in the form of small particles with $\mu$ size in order to make the surface-to-volume ratio large. It has been expected $^{3,4}$ that the finite size of the small particles may play some important roles for the heat flow.

In a previous paper, ${ }^{5}$ hereafter referred to as $I$, we have developed a theory of thermal boundary resistance between small particles and liquid He II. The paper I was an extension of the Khalatnikov's acoustic mismatch theory ${ }^{6}$ by allowing the finite size of solids, in which the heat transfer was assumed to be due to phonon conduction and the energy is carried away in the form of sound waves in liquid He II. The theory developed in I may be applicable to not only the case for liquid $\mathrm{He}$ II but also that for normal liquid ${ }^{3} \mathrm{He}$ in the hydrodynamic regime. In such a temperature regime, only the first sound plays as a collective mode. Below about $0.1 \mathrm{~K}$, where the Landau theory is known to be applicable to liquid ${ }^{3} \mathrm{He}$, zero sounds propagate as the collective modes in normal liquid ${ }^{3} \mathrm{He}$ and the heat may be carried by longitudinal and transverse zero sound and singlequasiparticle excitations.

The purpose of the present paper is to develop a theory of thermal boundary resistance between small particles and normal liquid ${ }^{3} \mathrm{He}$ below about $0.1 \mathrm{~K}$ in terms of Landau Fermi-liquid theory. ${ }^{7}$ This paper is organized as follows; in Sec. II, Landau Fermi-liquid theory is briefly reviewed in terms of spherical coordinates which make it easy to treat the energy emission from a spherical small particle. In Sec. III, the general expression for the heat conductance from a small particle into liquid ${ }^{3} \mathrm{He}$ is given. The eigenmodes of waves in a spherical particle are described in detail. In addition, these waves are quantized in this section. In Sec. IV, the numerical results of the thermal resistances are presented for the cases of copper and silver particles. Summary and discussions are given in Sec. $\mathrm{V}$.

\section{KINETIC EQUATION AND EMISSION OF ZERO SOUND FROM A SPHERICAL PARTICLE}

Theoretical attempts for the thermal boundary resistance between normal liquid ${ }^{3} \mathrm{He}$ and a bulk solid have been made by Bekarevich and Khalatnikov ${ }^{8}$ and Gavoret ${ }^{9}$ in terms of Landau Fermi-liquid theory. Recently Toombs et al. ${ }^{10}$ justified these phenomenological theory ${ }^{8,9}$ from the microscopic viewpoint. Although the transverse zero sound has not been considered in these works, it has been pointed out by Fomin ${ }^{11}$ that the transverse zero sound contributes to the heat flow. In this section, we describe briefly the kinetic equation of Fermi-liquid theory in terms of spherical coordinates.

The kinetic equation is expressed as

$$
\frac{\partial n_{\vec{p} \sigma}}{\partial t}+\frac{\partial \epsilon_{\vec{p} \sigma}}{\partial \vec{p}} \cdot \frac{\partial n_{\vec{p} \sigma}}{\partial \vec{r}}-\frac{\partial \epsilon_{\vec{p} \sigma}}{\partial \vec{r}} \cdot \frac{\partial n_{\vec{p} \sigma}}{\partial \vec{p}}=I\left[n_{\vec{p} \sigma}\right] \text {, }
$$


where $n_{\vec{p} \sigma}$ is the quasiparticle distribution function with a momentum $\overrightarrow{\mathrm{p}}$ and a spin $\sigma . \epsilon_{\overrightarrow{\mathrm{p}} \sigma}$ is the quasiparticle excitation energy and $I\left[n_{\vec{p} \sigma}\right]$ is the collision integral. For convenience, we suppress the spin in$\operatorname{dex} \sigma$ using the definition $\overrightarrow{\mathrm{p}}=\{\overrightarrow{\mathrm{p}}, \boldsymbol{\sigma}\}$.

Hereafter we concentrate on the collisionless regime because the presence of the finite lowest frequency emitted from the small particle of $\mu$ size leads to the condition, ${ }^{7} \omega \tau \gg>1$, in the temperature regime $(T<100 \mathrm{mK})$ considered in the present work. In this regime, Eq. (2.1) is rewritten by introducing the function $\nu_{\vec{p}}$ defined by $\delta n_{\vec{p}} \equiv-\left(\partial n \frac{0}{\vec{p}} / \partial \epsilon_{\vec{p}}\right) \nu_{\vec{p}}$;

$$
\frac{\partial}{\partial t} \nu_{\vec{p}}+\vec{v}_{\vec{p}} \cdot \frac{\partial}{\partial \vec{r}}\left(\nu_{\vec{p}}+\delta \epsilon_{\vec{p}}\right)=0 \text {. }
$$

Here $\vec{v}_{\vec{p}}$ is the quasiparticle velocity and $\delta \epsilon_{\vec{p}}$ is the variation of $\epsilon_{\vec{p}}$ produced from a change of the distribution function. The variation of energy can be written as

$$
\delta \epsilon_{\overrightarrow{\mathrm{p}}}=\epsilon_{\overrightarrow{\mathrm{p}}}-\epsilon_{\overrightarrow{\mathrm{p}}}^{0}=\frac{1}{V} \sum_{\overrightarrow{\mathrm{p}}^{\prime}} f_{\overrightarrow{\mathrm{p}} \overrightarrow{\mathrm{p}}^{\prime}} \delta n_{\overrightarrow{\mathrm{p}}^{\prime}},
$$

where $f_{\overrightarrow{\mathbf{p}} \overrightarrow{\mathrm{p}}^{\prime}} / V$ is the interaction energy between quasiparticles. The summation is replaced hereafter by the integral as defined by

$$
\underset{\overrightarrow{\mathrm{p}}}{\sum_{2}} \equiv \frac{V}{(2 \pi \hbar)^{3}} \sum_{\sigma} \int d^{3} p
$$

Equation (2.3) may be expanded in terms of the Landau parameters such as

$$
\delta \epsilon_{\vec{p}}=F_{0}^{s} \nu_{0}+F_{1}^{s} \vec{\nu}_{1} \cdot \hat{p}
$$

where $\hat{p}=\overrightarrow{\mathrm{p}} /|\overrightarrow{\mathrm{p}}|$. The moments $\nu_{0}$ and $\vec{\nu}_{1}$ are given by the following integral with respect to solid angle $\Omega_{\vec{p}}$

$$
\nu_{0}=\int \nu_{\overrightarrow{\mathrm{p}}} \frac{d \Omega_{\overrightarrow{\mathrm{p}}}}{4 \pi}, \quad \vec{\nu}_{1}=\int \nu_{\overrightarrow{\mathrm{p}}} \hat{p} \frac{d \Omega_{\overrightarrow{\mathrm{p}}}}{4 \pi} .
$$

Now let us consider a spherical elastic particle in liquid ${ }^{3} \mathrm{He}$ whose surface is executing vibrations. These vibrations act as a periodic perturbation to liquid ${ }^{3} \mathrm{He}$ and cause the change in the distribution function as

$\nu_{\overrightarrow{\mathrm{p}}}(\overrightarrow{\mathrm{q}}, \overrightarrow{\mathrm{r}})=\nu_{\overrightarrow{\mathrm{p}}}(q) \frac{R}{r} \exp [i(q r-\omega t)] f(\theta, \psi)$,

where $R$ is the radius of the particle and $\overrightarrow{\mathbf{r}}$ denotes the position vector from the origin. $f(\theta, \psi)$ is the dimensionless function dependent on the polar $(\theta)$ and azimuthal $(\psi)$ angle. Substituting Eq. (2.6) into Eqs. (2.2) and (2.3), we have the equation for $\nu_{\overrightarrow{\mathrm{p}}}(q)$ at a distance far from the origin,

$$
-s \nu_{\overrightarrow{\mathrm{p}}}+\mu\left(\nu_{\overrightarrow{\mathrm{p}}}+F_{0}^{s} \nu_{0}+F_{1} \vec{\nu}_{1} \cdot \hat{p}\right)=0,
$$

where $\mu=\hat{p} \cdot \hat{q}=\cos \theta_{p q} . \quad s$ is the dimensionless velocity of a collective mode defined by $s=\omega / v_{f} q . \quad v_{f}$ is the Fermi velocity of the quasiparticle. It should be noted here that the direction of $\vec{q}$ is parallel to that of $\vec{r}$ due to the spherical change of the distribution function. From Eq. (2.7), $\nu_{\vec{p}}$ becomes

$$
\nu_{\overrightarrow{\mathrm{p}}}=\frac{\mu}{s-\mu}\left(F_{0}^{s} \nu_{0}+F_{1}^{s} \vec{\nu}_{1} \cdot \hat{p}\right) .
$$

The scalar product in Eq. (2.7) is rewritten in terms of the column and row vector representations,

$$
\vec{\nu}_{1} \cdot \hat{p}=\left(\nu_{1 r}, \nu_{1 \theta}, \nu_{1 \psi}\right)\left(\begin{array}{c}
\mu \\
\left(1-\mu^{2}\right)^{1 / 2} \cos \psi_{p q} \\
\left(1-\mu^{2}\right)^{1 / 2} \sin \psi_{p q}
\end{array}\right) \text {, }
$$

where the azimuthal angle $\psi_{p q}$ is measured from the polar axis parallel to $\vec{q}$. The dispersion relation of longitudinal zero sound is obtained by integrating Eq. (2.8) over the solid angle, which yields

$$
\nu_{0}=\frac{F_{1}^{s} w(s) s}{1-w(s) F_{0}^{s}} \nu_{1 r},
$$

where

$$
w(s)=-1-\frac{1}{2} s \ln \left(\frac{s-1}{s+1}\right) .
$$

The number conservation law is derived by integrating Eq. (2.6)

$$
s \nu_{0}=\left(1+\frac{1}{3} F_{1}^{s}\right) \nu_{1 r} \text {. }
$$

By combining Eqs. (2.10) and (2.11), we have the dispersion relation of the longitudinal zero sound,

$$
w\left(s_{l}\right)=\left[F_{0}^{s}+F_{1}^{s} s_{l}{ }^{2} /\left(1+\frac{1}{3} F_{1}^{s}\right)\right]^{-1} .
$$

The dispersion relation of transverse zero sound is also derived by multiplying $\hat{p}$ to both hand sides of Eq. (2.2) and integrating with respect to momentum:

$$
w\left(s_{t}\right)=\frac{2}{1-s_{t}^{2}}\left(\frac{1}{F_{1}^{s}}-\frac{1}{6}\right) \text {. }
$$

In results, we have the change of the distribution function due to the surface vibrations of a spherical particle as

$$
\begin{aligned}
& \begin{array}{l}
\nu_{\overrightarrow{\mathrm{p}}}= \\
\quad \times \frac{\mu}{s_{l}-\mu}\left(F_{0}^{s} \nu_{0}+F_{1}^{s} \mu \nu_{1 r}\right)+\frac{\mu}{s_{t}-\mu} F_{1}^{s}\left(1-\mu^{2}\right)^{1 / 2} \\
\quad \times\left(\cos \psi_{p q} \nu_{1 \theta}+\sin \psi_{p q} \nu_{1 \psi}\right)
\end{array} \\
& \text { III. HEAT TRANSFER FROM A SPHERICAL } \\
& \text { PARTICLE INTO NORMAL LIQUID }{ }^{3} \mathrm{He}
\end{aligned}
$$

\section{HEAT TRANSFER FROM A SPHERICAL PARTICLE INTO NORMAL LIQUID ${ }^{3} \mathrm{He}$}

\section{A. Energy current}

The vibrating surface of a spherical particle gives rise to excitations in liquid ${ }^{3} \mathrm{He}$ as described in Sec. II 
and the excitations carry away the energy from the small particle. Energy current takes the form in terms of the Landau theory

$$
\overrightarrow{\mathrm{Q}}=\int \epsilon_{\overrightarrow{\mathrm{p}}} \frac{\partial \epsilon_{\overrightarrow{\mathrm{p}}}}{\partial \overrightarrow{\mathrm{p}}}\left[\delta n_{\overrightarrow{\mathrm{p}}}-\left(\frac{\partial n_{\overrightarrow{\mathrm{p}}}^{0}}{\partial \epsilon_{\overrightarrow{\mathrm{p}}}}\right) \delta \epsilon_{\overrightarrow{\mathrm{p}}}\right] d \tau \text {. }
$$

Here

$$
\int d \tau=\sum_{\sigma} V /(2 \pi \hbar)^{3} \int d^{3} p
$$

The quasiparticle energy $\epsilon_{\vec{p}}$ is expressed as $\epsilon_{\vec{p}}=\epsilon_{\vec{p}}^{0}$ $+\delta \epsilon_{\vec{p}}$ and $\epsilon_{\vec{p}}^{0}=v_{f}\left(p-p_{f}\right)+\mu_{c} . \quad p_{f}$ denotes the Fermi momentum and $\mu_{c}$ is the chemical potential of the quasiparticle. Then, we have the energy current $\overrightarrow{\mathrm{Q}}$ expressed as

$$
\begin{aligned}
\overrightarrow{\mathrm{Q}}= & -v_{f} \int \hat{p} \epsilon_{\overrightarrow{\mathrm{p}}}^{0}\left(\frac{\partial n_{\overrightarrow{\mathrm{p}}}^{0}}{\partial \epsilon_{\overrightarrow{\mathrm{p}}}}\right)\left(\nu_{\overrightarrow{\mathrm{p}}}+\delta \epsilon_{\overrightarrow{\mathrm{p}}}\right) d \tau \\
& -v_{f} \int \hat{p} \delta \epsilon_{\overrightarrow{\mathrm{p}}}\left(\frac{\partial{ }_{\overrightarrow{\mathrm{p}}}^{0}}{\partial \epsilon_{\overrightarrow{\mathrm{p}}}}\right)\left(\nu_{\overrightarrow{\mathrm{p}}}+\delta \epsilon_{\overrightarrow{\mathrm{p}}}\right) d \tau .
\end{aligned}
$$

The first term of the right-hand side of Eq. (3.2) vanishes because of the parity of the integrand. Performing the integral of the second term, we have the expression for $\overrightarrow{\mathrm{Q}}$ in terms of moments $\nu_{0}$ and $\vec{\nu}_{1}$

$\overrightarrow{\mathrm{Q}}=v_{f} N(0)\left\{\left(1+\frac{2}{3} F_{1}^{s}\right) F_{0}^{s} \nu_{0} \vec{\nu}_{1}+F_{1}^{s} \vec{\nu}_{1} \cdot\left[\nu_{2}\right]\right\}$,

where $N(0)$ is the density of quasiparticle states at the Fermi surface defined as $N(0)=m^{*} p_{f} /\left(\pi^{2} \hbar^{3}\right)$. The second-order moment $\left[\nu_{2}\right]_{i j}$ is defined by the relation

$$
\left[\nu_{2}\right]_{i j}=\int(\hat{p})_{i}(\hat{p})_{j} \nu_{\overrightarrow{\mathrm{p}}} d \Omega_{\overrightarrow{\mathrm{p}}} / 4 \pi .
$$

As far as it is concerned with the spherical zero sound, it is sufficient to take into account the radial component of energy current $\vec{Q}$ such as

$$
Q_{r}=v_{f} N(0)\left(F_{0}^{s}\left(1+\frac{2}{3} F_{1}^{s}\right) \nu_{0} \nu_{1 r}+F_{1}^{s} \sum_{i=r, \theta, \psi} \nu_{1 i} \nu_{2 r i}\right) \text {. }
$$

The momentum conservation law derived from Eq.

(2.2) takes the form in the column vector representation,

$$
\left(\begin{array}{l}
s_{t} \nu_{1 r} \\
s_{t} \nu_{1 \theta} \\
s_{t} \nu_{1 \psi}
\end{array}\right)=\left(\begin{array}{l}
\nu_{2 r r} \\
\nu_{2 r \theta} \\
\nu_{2 r \psi}
\end{array}\right)+\frac{1}{3} F_{0}^{s} \nu_{0}\left(\begin{array}{l}
1 \\
0 \\
0
\end{array}\right) \text {. }
$$

Substituting Eqs. (2.11) and (3.6) into Eq. (3.5), we have

$$
Q_{r}=v_{f} N(0)\left[F_{0}^{s} s_{l} \nu_{0}^{2}+F_{1}^{s} s_{l} \nu_{1 r}^{2}+F_{1}^{s} s_{t}\left(\nu_{1 \theta}^{2}+\nu_{1 \psi}^{2}\right)\right] .
$$

The fluid velocity $\vec{v}$ and the number density fluctua- tion $\delta n$ are related with the moments $\nu_{0}$ and $\vec{\nu}_{1}$ as follows:

$$
\overrightarrow{\mathrm{v}}=N(0) v_{f}\left(1+\frac{1}{3} F\{) \vec{\nu}_{1}\right.
$$

and

$$
\delta n=N(0) \nu_{0} \text {. }
$$

From Eqs. (3.8) and (3.9) the heat current $Q_{r}$ is rewritten as

$$
\begin{aligned}
Q_{r}= & \frac{1}{N(0)} F_{0}^{s} s_{l} v_{f}(\delta n)^{2} \\
& +\frac{F_{1}^{s}}{N(0) v_{f}^{2}\left(1+\frac{1}{3} F_{1}^{s}\right)^{2}}\left(s_{l} v_{f} v_{r}^{2}+s_{t} v_{f} v_{\theta}^{2}+s_{t} v_{f} v_{\psi}^{2}\right) .
\end{aligned}
$$

By integrating Eq. (3.10) with respect to a closed surface surrounding a small particle, the averaged energy flux $\bar{E}$ becomes

$$
\bar{E}=\int Q_{r}(\overrightarrow{\mathrm{r}}) r^{2} d \Omega / 4 \pi R^{2} .
$$

The energy currents $\vec{Q}$ at the respective positions $\vec{r}$ and $\overrightarrow{\mathrm{R}}$, are related to each other through; $Q_{r}(\overrightarrow{\mathrm{r}})$ $=Q_{r}(\overrightarrow{\mathrm{R}}) R^{2} / r^{2}$. Then we have the averaged energy flux $\bar{E}$ as

$$
\bar{E}=\int Q_{r}(\overrightarrow{\mathrm{R}}) d \Omega / 4 \pi .
$$

In I, we adopted the boundary condition that the normal component of fluid velocity near the surface is equal to that of the displacement velocity at surface. Because of the presence of viscosity in normal liquid ${ }^{3} \mathrm{He}$, the appropriate boundary condition may be the nonslip condition; all components of fluid velocity near the surface equal those of the surface velocity of spherical particle. Thus, we put the condition that the fluid near the moving surface oscillates in phase with the surface motion of spherical particle. The condition is expressed as

$$
n \frac{\partial \overrightarrow{\mathrm{u}}}{\partial t}=\overrightarrow{\mathrm{v}}
$$

where $\partial_{t} \overrightarrow{\mathrm{u}} \equiv\left\{\dot{u}_{r}, \dot{u}_{\theta}, \dot{u}_{\psi}\right\}$ is the time derivative of the surface displacement of a spherical particle. $\vec{v}$ is the fluid velocity near the surface. $n$ is the number density of liquid ${ }^{3} \mathrm{He}$ which is taken to be the global averaged value. Using Eqs. (2.11), (3.10), and (3.12), the energy flux $\bar{E}$ is expressed as

$$
\bar{E}=a_{1} \rho_{L} c_{l} \int \dot{u}_{r}^{2} d \Omega / 4 \pi+a_{2} \rho_{L} c_{t} \int\left(\dot{u}_{\theta}^{2}+\dot{u}_{\psi}^{2}\right) d \Omega / 4 \pi .
$$

Here the prefactors $a_{1}$ and $a_{2}$ are defined by

$$
\begin{aligned}
& a_{1}=F_{0}^{s} \rho_{L} /\left[N(0) c_{l}^{2}\left(m^{*}\right)^{2}\right]+a_{2}, \\
& a_{2}=F_{1}^{s} \rho_{L} /\left[N(0) p_{f}^{2}\right],
\end{aligned}
$$


where $\rho_{L}$ is the mass density of liquid ${ }^{3} \mathrm{He}$; $\rho_{L}=n m_{\mathrm{He}}$, and $c_{l}$ and $c_{t}$ are the velocities of longitudinal and transverse zero sound; $c_{l}=s_{l} v_{f}$ and $c_{t}=s_{t} v_{f}$, respectively.

\section{B. Surface vibrations of a spherical particle}

In order to estimate the energy flux $\dot{W}(T)$ from a small particle at temperature $T$ into liquid ${ }^{3} \mathrm{He}$, we must know the phonon states in a particle. The properties of phonons in a small particle have been described in I. Phonons in a spherical body consist of both the toroidal and spheroidal mode. We have also presented in I the quantized form of the spheroidal mode which contributes to the heat transfer into liquid $\mathrm{He}$ II. Here we shall give briefly the procedure of the quantization of toroidal mode.

The elastic wave equation in a spherical particle for the displacement $\overrightarrow{\mathrm{u}}(\overrightarrow{\mathrm{r}}, t)$ is written by Eq. (3.1) in $\mathrm{I}$. The solution $\overrightarrow{\mathrm{u}}$ for this equation can be expanded in terms of the potential fields

$$
\overrightarrow{\mathrm{u}}=\vec{\nabla} \psi_{1}+\vec{\nabla} \times \vec{\psi}_{2}+\vec{\nabla} \times \vec{\nabla} \times \vec{\psi}_{3},
$$

where the vector potentials $\vec{\psi}_{2}$ and $\vec{\psi}_{3}$ are defined as

$$
\vec{\psi}_{i}=(r, 0,0) \psi_{i}, \quad i=2,3 .
$$

Now let us consider the small particles in contact with liquid ${ }^{3} \mathrm{He}$. Since liquid ${ }^{3} \mathrm{He}$ has a small mass density $\left(\rho_{L} \sim 0.0815 \mathrm{~g} \mathrm{~cm}^{-3}\right)$ compared with that of small particles, the appropriate boundary conditions determining the eigenmodes may be taken as those for a stress-free surface. This condition allows us two types of oscillations in a spherical particle as described in I. One is the spheroidal mode and the other is the toroidal mode. The displacement vector belonging to spheroidal mode is expressed by the first and third term in Eq. (3.16). The displacement vector due to toroidal mode is expressed by the term $\vec{\nabla} \times \vec{\psi}_{2}$ in Eq. (3.16). It should be noted here that the spheroidal mode contributes to dilatation. The eigenvalue problem for the toroidal mode is described in the Appendix. In I, we have taken only the spheroidal mode into account for the heat flow because the transverse sound does not propagate in liquid $\mathrm{He}$ II. In the collisionless regime of liquid ${ }^{3} \mathrm{He}$, the contribution from the transverse zero sound, in addition to longitudinal one, plays an important role for the heat transfer. ${ }^{11}$ The spheroidal mode is expressed by the sum

$$
\overrightarrow{\mathrm{u}}_{s}^{J}=A_{s}^{J}\left(\vec{\nabla} \psi_{1}+\alpha_{J} \vec{\nabla} \times \vec{\nabla} \times \vec{\psi}_{3}\right),
$$

and the toroidal mode is

$$
\overrightarrow{\mathrm{u}}_{t}^{J}=A_{t}^{J} \vec{\nabla} \times \vec{\psi}_{2},
$$

where the lower suffices $s$ and $t$ mean the spheroidal and toroidal mode, respectively. The upper $J$ acts for a set of quantum numbers specified by $(l, m, \omega)$, where $l$ and $m$ give the order of spherical harmonics and $\omega$ is the angular frequency. Here $A_{s}^{J}$ and $A_{t}^{J}$ are the normalization factors for each mode. Although the first and second term on the right-hand side of Eq. (3.18) are actually independent of each other in a bulk solid, in the present cases those terms are combined into spheroidal modes owing to the boundary condition. The $\alpha_{J}$ in Eq. (3.18) indicates the ratio of the second to the first term, which is given by Eq.

(4.3) in I. These displacement fields, Eqs. (3.18) and (3.19), can be quantized by introducing creation and annihilation operator for bosons.

\section{Energy flux from a spherical particle at temperature $T$}

The energy flux $\dot{W}_{p}(T)$ at $T$ can be obtained by taking the thermal average of Eq. (3.14) and by summing up all over the modes as follows:

$$
\begin{aligned}
\dot{W}_{p}(T)= & a_{1} \rho_{L} c_{l} \sum_{J, \alpha=s, t} \int\left\langle\dot{u}_{\mathrm{op}, \alpha, r}^{J^{\dagger}}(\overrightarrow{\mathrm{R}}) \dot{u}_{\mathrm{op}, \alpha, r}^{J}(\overrightarrow{\mathrm{R}})\right\rangle_{T} d \Omega / 4 \pi \\
& +a_{2} \rho_{L} c_{t} \sum_{J, \alpha=s, t, \beta=\theta, \psi} \int\left\langle\dot{u}_{\mathrm{op}, \alpha, \beta}^{J^{\dagger}}(\overrightarrow{\mathrm{R}}) \dot{u}_{\mathrm{op}, \alpha, \beta}^{J}(\overrightarrow{\mathrm{R}})\right\rangle_{T} d \Omega / 4 \pi,
\end{aligned}
$$

where the angle brackets denote the thermal average at $T$. The thermally averaged squared displacement can be written explicitly in terms of the Bose-Einstein distribution function $n_{B}(\omega, T)$ as

$$
\left\langle\overrightarrow{\mathrm{u}}_{\mathrm{op}, \alpha}^{J \dagger} \overrightarrow{\mathrm{u}}_{\mathrm{op}, \alpha}^{J}\right\rangle_{T}=\frac{\hbar}{\rho_{S} \omega_{J}} n_{B}\left(\omega_{J}, T\right)\left|\overrightarrow{\mathrm{u}}_{\alpha}^{J}\right|^{2}
$$

Substituting the expression for $u_{t}^{J}$ and $u_{s}^{J}$ into Eq. (3.21) we have the heat flux given by

$$
\dot{W}_{p}(T)=\frac{\rho_{L}}{4 \pi \rho_{S}} \sum_{l, \omega_{J}}(2 l+1) \hbar \omega_{J} n_{B}\left(\omega_{J}, T\right)\left\{a_{1} c_{l}\left(A_{s}^{J}\right)^{2} G_{s r}^{J}(R)+a_{2} c_{t}\left[\left(A_{s}^{J}\right)^{2} G_{s \theta \psi}^{J}(R)+\left(A_{t}^{J}\right)^{2} G_{t}^{J}(R)\right]\right\} .
$$


The normalization factors $A_{s}^{J}$ and $A_{t}^{J}$ are related to $G_{s r}^{J}, G_{s \theta \psi}^{J}$, and $G_{t}^{J}$ through

$$
\begin{aligned}
& A_{s}^{J}=\left(\int_{0}^{R} d r r^{2}\left(G_{s r}^{J}+G_{s \theta \psi}^{J}\right)\right)^{-1 / 2}, \\
& A_{t}^{J}=\left(\int_{0}^{R} d r r^{2} G_{t}^{J}\right)^{-1 / 2} .
\end{aligned}
$$

Here we have defined,

$$
\begin{aligned}
& G_{s r}^{J}(r)= {\left[\partial_{r} j_{l}\left(\omega r / v_{s}\right)\right]^{2} } \\
&+\left[\alpha(l) l(l+1) j_{l}\left(\omega r / v_{t}\right) r^{-1}\right]^{2}, \\
& G_{s \theta \psi}^{J}(r)=l(l+1)\left[j_{l}^{2}\left(\omega r / v_{s}\right) r^{-2}\right. \\
&\left.+\alpha^{2}(l) j_{l}^{2}\left(\omega r / v_{t}\right)\right],
\end{aligned}
$$

and

$$
G_{t}^{J}(r)=j_{l}^{2}\left(\omega r / v_{t}\right)
$$

where $v_{s}$ and $v_{t}$ are the velocities of spheroidal and toroidal waves, respectively; $v_{s}=\left[(2 \mu+\lambda) / \rho_{S}\right]^{1 / 2}$ and $v_{t}=\left(\mu / \rho_{S}\right)^{1 / 2}$, and $\lambda$ and $\mu$ are Lamé coefficients. The explicit form of $\alpha(l)$ is given as

$$
\alpha(l)=\frac{2(l-1) j_{l}(\xi)-2 \xi j_{l+1}(\xi)}{\left[\eta^{2}-2\left(l^{2}-1\right)\right] j_{l}(\eta)-2 \eta j_{l+1}(\eta)},
$$

where $\xi=\omega R / v_{s}$ and $\eta=\omega R / v_{t}$. In Eq. (3.22), the summation is carried out with respect to $l$ and $\omega_{\mathrm{J}}$.
The prefactor $(2 l+1)$ in Eq. (3.22) denotes the degeneracy due to the sum over the quantum number $m$.

\section{NUMERICAL RESULTS FOR $R_{K}$ BETWEEN METALLIC PARTICLES AND LIQUID ${ }^{3} \mathrm{He}$}

Let us consider the heat flow from a small particle at temperature $T+\Delta T$ into liquid ${ }^{3} \mathrm{He}$ at $T$. The net heat flux across the interface is given by

$$
\Delta \dot{W}_{p}(T)=\dot{W}_{p}(T+\Delta T)-\dot{W}_{L}(T),
$$

where $\dot{W}_{p}(T+\Delta T)$ is the heat flux into liquid ${ }^{3} \mathrm{He}$ when a particle is maintained at temperature $T+\Delta T$ and $\dot{W}_{L}(T)$ is the heat flux from liquid ${ }^{3} \mathrm{He}$ into small particle at $T$. Since the net heat flux has to vanish at equal temperature $(\Delta T=0)$, we have the relation $\dot{W}_{L}(T)=\dot{W}_{p}(T)$. If the difference in temperature $\Delta T$ is small, Eq. (4.1) becomes

$$
\Delta \dot{W}_{p}(T)=h_{K} \Delta T \text {, }
$$

where the heat conductance $h_{K}$ is defined by

$$
h_{K}=\frac{\partial}{\partial T} \dot{W}_{p}(T)
$$

The thermal resistance $R_{K}$ is the inverse of $h_{K}$, i.e., $R_{K}=h_{K}^{-1}$. From Eq. (4.3), we have the expression for the conductance as

$$
h_{K}=\frac{\rho_{L} \hbar}{4 \pi \rho_{S}} \sum_{l, \omega_{J}}(2 l+1) \omega_{J} \partial_{T} n_{B}\left(\omega_{J}, T\right)\left\{a_{1} c_{l}\left(A_{s}^{J}\right)^{2} G_{s r}^{J}(R)+a_{2} c_{t}\left[\left(A_{s}^{J}\right)^{2} G_{s \theta \psi}^{J}(R)+\left(A_{t}^{J}\right)^{2} G_{t}^{J}(R)\right]\right\} \text {. }
$$

Now we can estimate the resistance $R_{K}$ using the formula (4.4). We need the numerical factor $a_{1}$ and $a_{2}$ and the velocities of zero sounds $c_{l}$ and $c_{t}$ in Eq. (4.4) from the Landau parameters $F_{0}^{s}$ and $F_{1}^{s}$. Provided that the parameters are quoted from Ref. 12, we obtain the numerical estimation such as $a_{1}=1.54$, $a_{2}=0.64 c_{l}=3.456 v_{f}$, and $c_{t} \cong v_{f}$, respectively. Let us consider the case of the heat transfer between liquid ${ }^{3} \mathrm{He}$ and copper or silver particles. The eigenvalue equation for the spheroidal mode in a small particle has been solved in I and shown in Table I of I. The eigenvalue equation for the toroidal mode [Eq. (A5) in the Appendix] is solved numerically in the present work, in which a set of parameters are taken as $v_{s}=5.01 \times 10^{5} \mathrm{~cm} / \mathrm{sec}$ and $v_{t}=2.27$ $\times 10^{5} \mathrm{~cm} / \mathrm{sec}$ for copper and $v_{s}=3.60 \times 10^{5} \mathrm{~cm} / \mathrm{sec}$ and $v_{t}=1.66 \times 10^{5} \mathrm{~cm} / \mathrm{sec}$ for silver particles, respectively. The mass densities are $\rho_{p}=8.96 \mathrm{~g} \mathrm{~cm}^{-3}$ for copper and $\rho_{p}=10.5 \mathrm{~g} \mathrm{~cm}^{-3}$ for silver. All of these parameters are taken from the corresponding bulk values. The numerical results on the eigenvalues of the toroidal modes for copper particle are given in Table I of the Appendix up to $l=10$. It should be noted that the lowest eigenvalue for the toroidal mode $\left(\omega_{t}^{0}=1.14 \times 10^{10} \mathrm{sec}^{-1}\right)$ is slightly larger than that of the spheroidal mode $\left(\omega_{s}^{0}=1.11 \times 10^{10} \mathrm{sec}^{-1}\right)$ for a copper particle $1 \mu \mathrm{m}$ in diameter. It will be seen from Table I that the number of states per unit interval of $\eta$ increases with approaching the larger part of eigenvalues. The reason of this tendency is identical with that for the spheroidal mode, i.e., the wavelengths at large eigenvalues are short compared with the size of a particle. Then the number of states in the region of large eigenvalues becomes denser.

A number of experiments ${ }^{13-25}$ have been made on the thermal resistances between metals and liquid ${ }^{3} \mathrm{He}$, in which the various sizes and shapes of metallic particles have been used. We have calculated the thermal resistance from Eq. (4.4) and compared it with the observed data. Figure 1 shows the calculated results of the thermal boundary resistances $R_{K}$ between liquid ${ }^{3} \mathrm{He}$ and silver particles with $R=0.55$ and $2.5 \mu \mathrm{m}$ in radii, where the eigenvalues up to $\eta=100$ are taken into account. We see from Fig. 1 that the resistances exhibit a $T^{-3}$ dependence above about $10 \mathrm{mK}$ for $R=0.55 \mu \mathrm{m}$ and $2 \mathrm{mK}$ for 


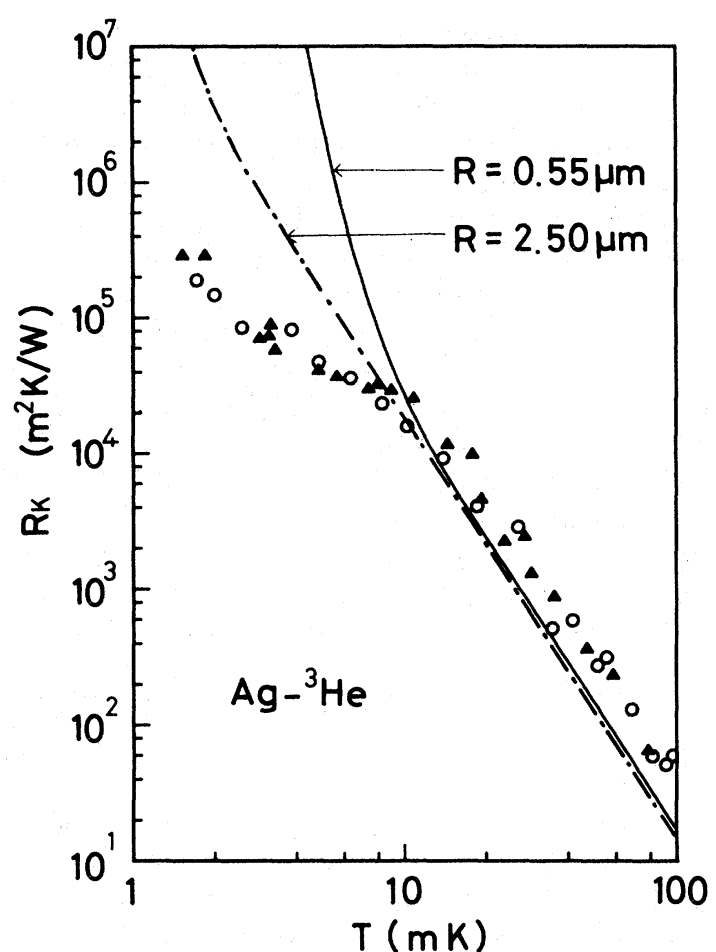

FIG. 1. The thermal boundary resistances as a function of temperature between liquid ${ }^{3} \mathrm{He}$ and silver particles with 0.55 and $2.5 \mu \mathrm{m}$ in radii. The curves give the contributions from both longitudinal and transverse zero sound. The observed data from Ref. 20 are given for dirty silver particles with $R=0.4 \mu \mathrm{m}$ in radius $(\Delta)$ and for clean silver particle with $R=0.55 \mu \mathrm{m}(\mathrm{O})$, respectively.

$R=2.5 \mu \mathrm{m}$. At low temperatures, the resistances $R_{K}$ increase exponentially with decreasing temperature. These exponential behavior may be caused by the size effect of small particles as explained in I.

The measured resistances for silver particles with $R=0.55 \mu \mathrm{m}$ are also plotted from Ref. 20 in Fig. 1 . The circles and triangles are the data of clean particles, including dilute magnetic impurities, and the dirty particles involving a lot of impurities, respectively. Above roughly $10 \mathrm{mK}$, the calculated resistances for $R=0.55 \mu \mathrm{m}$ agree well with the observed data in both magnitude and temperature dependence. At temperature less than roughly $10 \mathrm{mK}$, the observed resistances show closely to the $T^{-1}$ dependence and are about two or three orders of magnitude smaller than the calculated values. These discrepancies between theory and experiments may suggest the importance of other dominant mechanisms for heat exchange between metallic particles and liquid ${ }^{3} \mathrm{He}$.

The calculated resistances for copper particles with $R=0.5$ and $2.5 \mu \mathrm{m}$ are given in Fig. 2. The similar tendency on the temperature dependence of the resistances is found as in the case of silver particles. For

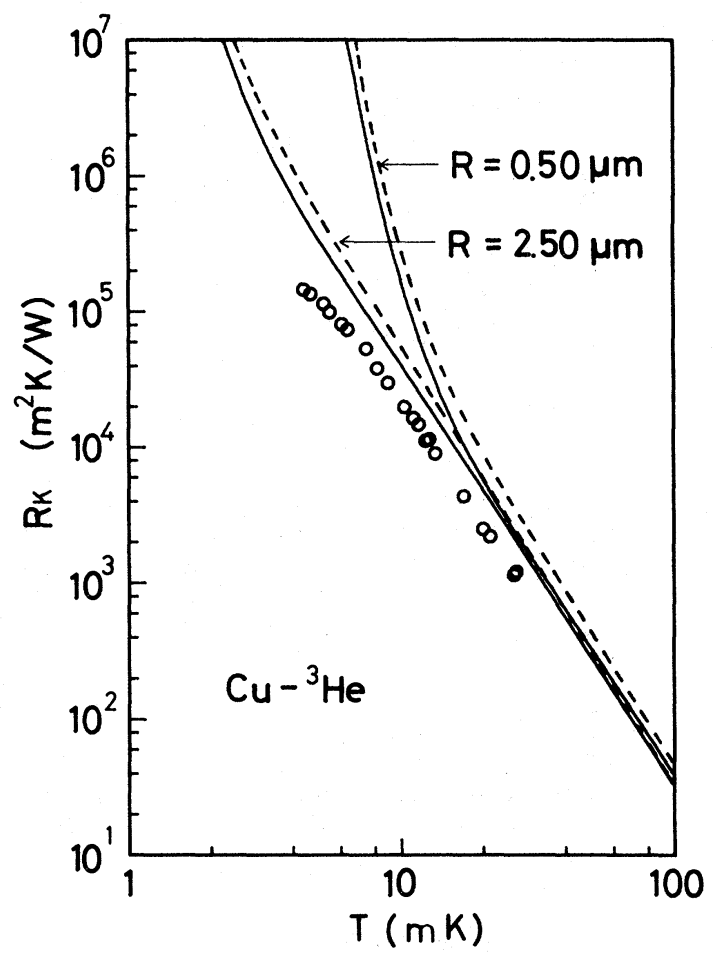

FIG. 2. The thermal boundary resistances $R_{K}$ vs temperature between liquid ${ }^{3} \mathrm{He}$ and copper particles with 0.5 and $2.5 \mu \mathrm{m}$ in radii. The solid curves exhibit the contributions from both the longitudinal and transverse zero sound, and the dashed curves are only because of longitudinal mode. The data from Ref. 17 for copper flakes (thickness $<1 \mu \mathrm{m}$ and diameter $\sim 30 \mu \mathrm{m})$ are shown by circles.

references, we also plot the measured resistance using copper flakes with thickness $(<1 \mu \mathrm{m})$ and diameter $(-30 \mu \mathrm{m})$ from Ref. 17. As seen from Fig. 2 , in the high-temperature regime $(T>10 \mathrm{mK})$, the agreement between theory and experiments is excellent as well as the case of silver particles.

The thermal resistances only due to longitudinal zero sound for copper particles are also given in Fig. 2. The contribution to the thermal conductance due to transverse zero sound is not negligible as shown in Fig. 2. We plot in Fig. 3 the relative contributions to the heat conductance due to longitudinal and transverse zero sound. The ordinate in Fig. 3 is taken to be $h_{K}$ (transverse or longitudinal zero sound) $/ h_{K}$ (total). As is seen from Fig. 3, the longitudinal zero sound excited by spheroidal mode plays a dominant role to the heat transfer throughout the temperature region. At very low temperatures, the conductance from the spheroidal mode into transverse zero sound almost vanishes because the lowest mode of spheroidal waves gives only the volume expansion and/or contraction and does not have the tangential component of the surface displacement. The heat transfer from toroidal mode to 


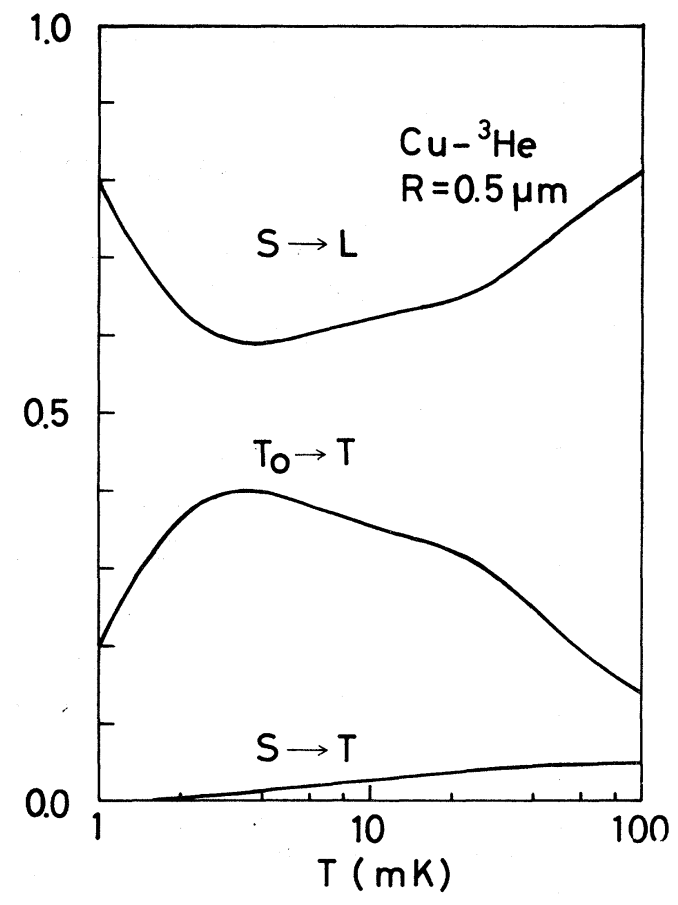

FIG. 3. The relative contributions to the heat conductance due to the longitudinal or transverse zero sound. $S$ and $T_{o}$ mean the spheroidal and toroidal mode, respectively. $L$ and $T$ indicate the longitudinal and transverse zero sound. The ordinate is defined as the ratio $h_{K}(S \rightarrow L, S \rightarrow T$, or $\left.T_{o} \rightarrow T\right) / h_{K}$ (total). The abscissa is the temperature.

transverse zero sound becomes comparable to that from spheroidal mode to longitudinal zero sound at around $3 \mathrm{mK}$. This originates from the difference in the lowest eigenvalues between spheroidal and toroidal mode.

\section{SUMMARY AND DISCUSSIONS}

We have studied the thermal boundary resistance $R_{K}$ between small particles and liquid ${ }^{3} \mathrm{He}$ in terms of Landau Fermi-liquid theory. The expression of the thermal resistance, Eq. (4.4), has been derived by illustrating a spherical particle with an arbitrary size. It has been shown that both the spheroidal and toroidal mode in a particle contribute to the heat transfer through the excitation of longitudinal and transverse zero sound in contrast to the case of liquid He II. The eigenvalues of the toroidal modes have been solved numerically from Eq. (A5) in the Appendix. Using these eigenvalues and those for spheroidal modes obtained in $\mathrm{I}$, the resistances $R_{K}$ have been calculated for silver and copper particles with various sizes in Figs. 1 and 2. At temperatures higher than those corresponding to the finite lowest eigenfrequency of small particles, the calculated curves in
Figs. 1 and 2 exhibit the $T^{-3}$ dependence and the magnitude is in agreement with the bulk limit. The physical meaning of these results is clear because the dominant phonons contributing to the heat transfer in this temperature regime have much shorter wavelengths than the size of particles, then the shape is irrelevant and the resistance $R_{K}$ will approach to bulk limit. The exponential behavior for the resistances at very low temperatures would be common for the various shapes of particles, because the presence of the lowest eigenfrequency in any particles with finite size is essential to this dependence. We have calculated in Fig. 3 the relative contributions to heat transfer due to longitudinal and transverse zero sound, respectively, and shown that the transverse zero sound contributes to the heat transfer in comparable degree with the transfer due to longitudinal zero sound.'

Andres and Sprenger ${ }^{20}$ have observed the resistance between silver particles $0.55 \mu \mathrm{m}$ in radius and liquid ${ }^{3} \mathrm{He}$. We have compared our theoretical results with their data in Fig. 1. As seen from Fig. 1, the agreement between theory and experiments is excellent above about $10 \mathrm{mK}$. At temperatures less than roughly $10 \mathrm{mK}$ the measured resistances ${ }^{20}$ become proportional to $T^{-1}$. This has been also found to be the case for the resistance between other metals and liquid ${ }^{3} \mathrm{He},{ }^{21}$ which may suggest that the unknown process transferring heat effectively is present. We have also compared the theory with the measured resistance $R_{K}$ for copper flakes ${ }^{17}$ in Fig. 2. At temperatures higher than roughly $10 \mathrm{mK}$ the calculated result agrees with the observed values on $R_{K}$ in both magnitude and temperature dependence. The measured resistances ${ }^{17}$ deviate from the calculated curves at very low temperatures as in the case of silver particles. At the present stage, the physical origin of these discrepancies is not clear and it seems to need further investigations on these points.

In conclusion, we have studied the thermal boundary resistance between small particles and liquid ${ }^{3} \mathrm{He}$ due to zero sound excitations by taking account of the size effect of small particles. The present results will provide useful physical insight into the further understanding for the problems.

\section{ACKNOWLEDGMENTS}

The authors are grateful to Professor T. Sakuma for helpful discussions.

\section{APPENDIX: EIGENVALUES OF TOROIDAL MODES}

Here, we shall solve the eigenvalues equation for the toroidal mode. Substituting Eq. (3.16) into the wave equation for the displacement vector [Eq. (3.1) 
TABLE I. The dimensionless eigenvalue $\eta$ of toroidal mode. The integer $l$ of the column means the order of Legendre polynomial. The integer $n$ of the row indicates the $n$th modes belonging to the $l$ th-order oscillation.

\begin{tabular}{|c|c|c|c|c|c|c|c|c|c|c|}
\hline & 1 & 2 & 3 & 4 & 5 & 6 & 7 & 8 & 9 & 10 \\
\hline 1 & $\ldots$ & 4.27 & 7.59 & 10.81 & 13.99 & 17.16 & 20.32 & 23.47 & 26.62 & 29.77 \\
\hline 2 & $\ldots$ & 5.76 & 9.09 & 12.32 & 15.51 & 18.68 & 21.85 & 25.01 & 28.16 & 31.32 \\
\hline 3 & 2.50 & 7.13 & 10.51 & 13.77 & 16.98 & 20.17 & 23.34 & 26.51 & 29.67 & 32.83 \\
\hline 4 & 3.86 & 8.44 & 11.88 & 15.17 & 18.41 & 21.61 & 24.80 & 27.98 & 31.15 & 34.32 \\
\hline 5 & 5.09 & 9.71 & 13.21 & 16.54 & 19.80 & 23.03 & 26.24 & 29.43 & 32.61 & 35.79 \\
\hline 6 & 6.26 & 10.95 & 14.51 & 17.88 & 21.18 & 24.43 & 27.65 & 30.86 & 34.05 & 37.24 \\
\hline 7 & 7.40 & 12.16 & 15.78 & 19.20 & 22.52 & 25.80 & 29.04 & 32.27 & 35.47 & 38.67 \\
\hline 8 & 8.51 & 13.36 & 17.04 & 20.50 & 23.86 & 27.15 & 30.42 & 33.66 & 36.88 & 40.08 \\
\hline 9 & 9.62 & 14.54 & 18.28 & 21.78 & 25.17 & 28.49 & 31.78 & 35.03 & 38.27 & 41.49 \\
\hline 10 & 10.71 & 15.72 & 19.51 & 23.05 & 26.47 & 29.82 & 33.12 & 36.39 & 39.64 & 42.88 \\
\hline
\end{tabular}

in I], we have the equation for the vector potential $\vec{\psi}_{2}$

$$
\rho_{S} \frac{\partial^{2}}{\partial t^{2}} \vec{\nabla} \times \vec{\psi}_{2}-\mu \nabla^{2} \vec{\nabla} \times \vec{\psi}_{2}=0
$$

where $\rho_{S}$ is the mass density of a small particle. Specifying the form of the vector potential $\psi_{2}$ as $\overrightarrow{\mathrm{r}} \psi_{2}$ and utilizing the identity that $\vec{\nabla} \times\left[\vec{r} \psi_{2}\right]=-\vec{r}$ $\times \vec{\nabla} \psi_{2}$, we derive the equation for the scalar function $\psi_{2}$;

$$
\overrightarrow{\mathrm{r}} \times \vec{\nabla}\left(\rho_{S} \ddot{\psi}_{2}-\mu \nabla^{2} \psi_{2}\right)=0
$$

Provided that there is no potential source in the elastic body, we have the scalar wave equation as

$$
\rho_{S} \ddot{\psi}_{2}-\mu \nabla^{2} \psi_{2}=0
$$

The displacement field due to the mode $(l, m)$ are derived using the definition of the vector potential $\vec{\psi}_{2}=(r, 0,0) \psi_{2}$ and Eq. (3.16),

$\left(\begin{array}{l}u_{r}^{l, m} \\ u_{\theta}^{l, m} \\ u_{\psi}^{l, m}\end{array}\right)=\left(\begin{array}{c}0 \\ -m A_{t}^{l, m} j_{l}\left(k_{2} r\right) P_{l}^{|m|}(\cos \theta) \sin m \psi / \sin \theta \\ A_{t}^{l, m} j_{l}\left(k_{2} r\right) \frac{\partial}{\partial \theta} P_{l}^{|m|}(\cos \theta) \cos m \psi\end{array}\right) e^{-i \omega t}$.

(A4)

Making use of the stress-free boundary conditions at the surface $(r=R)$, we have the eigenvalue equation for the toroidal mode for $l \geqslant 1$,

$$
j_{l+1}(\eta)-(l-1) j_{l}(\eta) / \eta=0 \text {. }
$$

We see from Eq. (A4) that the toroidal mode has no radial component. By solving numerically Eq. (A5), we have the eigenvalues as shown in Table I, in which the dimensionless eigenvalues $\eta=k_{2} R$ are tabulated up to $l=10$.
1P. L. Kapitza, J. Phys. (Moscow) 4, 181 (1941). This paper may also be found in Collected Papers of P. L. Kapitza, edited by D. ter Haar (McMillan, New York, 1964), Vol. II, p. 581.

${ }^{2}$ D. M. Lee and H. A. Fairbank, Phys. Rev. 116, 1359 (1959).

3J. P. Harrison and D. B. McColl, J. Phys. C 10, L297(1977).

4J. P. Harrison, J. Low Temp. Phys. 37, 467 (1979).

${ }^{5}$ T. Nakayama and N. Nishiguchi, Phys. Rev. B $\underline{24}, 6421$ (1981).

6I. M. Khalatnikov, Zh. Eksp. Theor. Fiz. 22, 687 (1952); and Introduction to the Theory of Superfluidity (Benjamin, New York, 1965), p. 138.

${ }^{7}$ For a recent review, see G. Baym and C. Pethik, The Physics of Liquid and Solid Helium (Wiley, New York, 1978), Part II, p. 1.

${ }^{8}$ I. L. Bekarevich and I. M. Khalatnikov, Zh. Eksp. Theor.
Fiz. 39, 1699 (1960) [Sov. Phys. JETP 12, 1187 (1961)].

9J. Gavoret, Phys. Rev. 137, A721 (1965).

${ }^{10}$ G. A. Toombs, F. W. Sheard, and M. J. Rice, J. Low Temp. Phys. 39, 273 (1980).

${ }^{11}$ I. A. Fomin, Zh. Eksp. Theor. Fiz. 54, 1881 (1968) [Sov. Phys. JETP 27, 1010 (1968)].

12J. C. Wheatley, Rev. Mod. Phys. 47, 415 (1975).

${ }^{13}$ W. R. Abel, A. C. Anderson, W. C. Black, and J. C. Wheatley, Physics (N.Y.) 1, 337 (1965).

${ }^{14}$ W. C. Black, A. C. Mota, J. C. Wheatley, J. H. Bishop, and P. M. Brewster, J. Low Temp. Phys. 4, 391 (1971).

${ }^{15}$ J. H. Bishop, D. W. Cutter, A. C. Mota, and J. C. Wheatley, J. Low Temp. Phys. 10, 379 (1973).

16J. H. Bishop, A. C. Mota, and J. C. Wheatley, in Proceedings of the 13th International Conference on Low Temperature Physics, edited by K. D. Timmerhaus et al. (Plenum, New York, 1974), Vol. 1, p. 406. 
${ }^{17}$ D. Avenel, M. P. Berglund, R. G. Gylling, N. E. Phillips, A. Vetsleseter, and M. Vuorio, Phys. Rev. Lett. 31,76 . (1973).

18J. M. Dundon, D. L. Stolfa, and J. M. Goodkind, Phys. Rev. Lett. 30, 843 (1973).

${ }^{19} \mathrm{R}$. Radebaugh and J. D. Siegwarth, in Proceedings of the 13th International Conference on Low Temperature Physics, edited by K. D. Timmerhaus et al. (Plenum, New York, 1974), Vol. 1, p. 401.

${ }^{20} \mathrm{~K}$. Andres and W. O. Sprenger, in Proceedings of the 14 th International Conference on Low Temperature Physics, edited by $M$. Krusius and M. Vuorio (North-Holland, Amster- dam, 1975), Vol. 1, p. 123.

${ }^{21}$ A. I. Ahonen, P. M. Berglund, M. T. Haikala, M. Krusius, O. V. Lounasmaa, and M. A. Paalanen, Cryogenics $\underline{16}$, 521 (1976).

${ }^{22}$ A. I. Ahonen, O. V. Lounasmaa, and M. C. Veuro, J. Phys. (Paris) 39, C6-265 (1978).

${ }^{23}$ E. Varoquaux, J. Phys. (Paris) 38, C6-1605 (1978).

${ }^{24}$ D. O. Edwards, J. D. Feder, W. J. Gully, G. G. Ihas, J. Landau, and K. A. Muelhing, J. Phys. (Paris) 39, C6-260 (1978).

${ }^{25}$ T. Kusumoto, T. Mizusaki, and A. Hirai, Physica (Utrecht) 108B, 919 (1981). 